\title{
Abstracts from the 2015 Research Poster Session at the American Massage Therapy Association Annual Convention
}

\section{A CASE STUDY UTILIZING MYOFASCIAL RELEASE, ACUPRESSURE, AND TRIGGER-POINT THERAPY TO TREAT BILATERAL "STRINGHALT" IN A 12-YEAR-OLD AKHAL-TEKE HORSE}

Tammy Brockman, MS, ATC/L, LMT, CLT, Stacey Powell, LMT

Introduction: "Stringhalt" is a horse condition that causes one or both hind legs to spasm when walking or trotting. The condition is thought to be related to a neurological cause from plant toxicity or peripheral nerve injury. The prognosis is poor, and the horse's performance and quality of life can be affected. Treatment has included surgically cutting the digital extensors, with varied results and additional problems.

Case Presentation: The case study is a 12-year-old Akhal-Teke horse of excellent pedigree. In 2011, she was caught in barbed wire overnight and sustained lacerations to the bone. Shortly after the injury, the horse was placed in an inadequate stall for several months and was unable to walk or run, developing stringhalt. Currently, her condition is aggravated by stress and dietary changes, and alleviated by certain types of massage (myofascial, acupressure, triggerpoint release). The incidence of stringhalt is every $3-5$ minutes, with more frequent and severe symptoms on the right. The horse is unable to run or back up.

Methods: Six 1 to 1.5 hour bi-weekly treatments were performed. The treatments consisted of myofascial release at the cervical, sacrum, and iliums; acupressure of the bladder meridian including c-spine, t-spine, L-spine, and hamstring; and trigger-point release of the iliacus.

Results: After six treatments, the horse was seen running and standing in a position that promotes hip extension. She had not been able to do either since the injury. The frequency and severity of the spasms have decreased to every 10-20 minutes. The horse's owners report that her disposition and quality of life are much improved.

Discussion: The results suggest that myofascial release, acupressure, and trigger-point therapy may be utilized to provide a positive treatment outcome in the case of stringhalt. Note that the scope of practice varies by state, and special training is needed to work with the equine population.

\section{HAND SELF-SHIATSU FOR SLEEP PROBLEMS IN PERSONS WITH CHRONIC PAIN: A PILOT STUDY*}

Cary A. Brown, PhD, Geoff Bostick, PhD, Leisa Bellmore, CST

Introduction: Difficulty falling asleep is a common problem for persons living with pain. Research demonstrates that disrupted sleep will, in turn, exacerbate the chronic pain problem. The evidence-base for a range of pragmatic, nonpharmacological sleep interventions that can potentially be incorporated into pain management programs is growing. However, strategies that are controlled by the patient and are congruent with the self-management model favored by most pain services are not yet well researched. This study looks at the outcome of teaching adults with enduring musculoskeletal pain a standardized, pre-bedtime, self-administered Shiatsu hand massage (SHM) intervention to promote sleep onset.

Method: A range of standardized sleep-related, self-report tools and objective sleep actigraphy (recorded for 5-7 nights) were used to collect baseline data. Participants were then taught pre-bedtime, self-administered SHM in one-to-one sessions. They also received two follow-up phone calls to offer support and clarification, if needed. The assessment battery and 5-7 nights of actigraphy data were collected again at 2 weeks and 8 weeks postSHM training.

Results: Twelve persons with diverse musculoskeletal pain experiences participated. Data collected at baseline, 2-week, and 8-week follow-up periods revealed no apparent changes in actigraphy scores. Treatment fidelity dropped off at 8-week follow-up. A trend toward improved self-reported sleep latency (time to fall asleep) and sleep duration (time spent asleep) emerged. A number of participants reported they were more concerned with increasing their period of unbroken sleep as opposed to their total sleep time. None of the participants reported adverse effects of the intervention.

Conclusion: Preliminary findings of a lowcost, pragmatic, patient-controlled intervention are

* Brown CA, Bostick G, Bellmore L, Kumanayaka D. Hand selfShiatsu for sleep problems in persons with chronic pain: a pilot study. J Integr Med. 2014;12(1):94-101. 
promising. Further study of self-administered SHM to determine the potential mechanism(s) at play, with greater control of treatment fidelity, and to investigate its use during night time awakenings in addition to pre-bedtime, are particularly indicated.

Support: Canadian CAM Research Fund (CCRF)

\section{ACUPUNCTURE AND MASSAGE FOR PAIN RELIEF OF FAMILIAL MEDITERRANEAN FEVER IN PEDIATRIC PATIENTS: A CLINICAL CASE REPORT}

Edward Cho, BA, BS, Marie Lee, BS, Rika Meyer, PhD

Background: Familial Mediterranean Fever (FMF) is an autosomal recessive disease characterized by intractable pain due to serosal inflammation and sporadic recurring fevers. Little research currently exists on alternative, nonpharmacological interventions for pain management of FMF.

Purpose: In this case report, we examine the effect of acupuncture and massage on chronic pain associated with FMF in 2 pediatric patients.

Methods: Patient \#1, an 11-year-old Hispanic male, and patient \#2, an 8-year-old Caucasian female, are FMF patients with diagnoses of chronic pain. Patients received 6 weeks of therapy (acupuncture and/or massage) followed by a 4-week treatment gap. Patient \#1 received acupuncture followed by massage, and patient \#2 received massage only. Each treatment included a $30-\mathrm{min}$ pretest and a $10-\mathrm{min}$ post-test. Surveys assessed pain, physical health, and psychosocial health per the Pediatric Quality of Life Inventory (PedsQL), Brief Pain Inventory (BPI), Visual Analogue Scales (VAS), and FACES Pain Scale.

Results: Both patients showed immediate improvements in VAS and FACES pain measures following treatment with a total or near total relief of pain observed in the majority of recorded instances where patients presented with pain. Physical health scores showed a slight worsening in patient \#1, but a moderate improvement in patient \#2. BPI pain severity scores slightly worsened for patient \#1 but improved for patient \#2, while BPI pain interference scores slightly improved for patient \#1 and slightly worsened for patient \#2.

Conclusions: Acupuncture and massage may be a promising palliative treatment for chronic pain associated with FMF. However, findings are limited due to small sample size and lack of baseline data (week 1 pretest data) for patient \#2. Future research should conduct a larger RCT on acupuncture and massage for pediatric FMF patients and other chronic pain conditions, such as fibromyalgia, systemic lupus erythematosus, and rheumatoid arthritis.

\section{REGIONAL ANALYSIS OF MASSAGE THERAPY IN OUTPATIENT CANCER CENTERS}

Virginia S. Cowen, PhD, LMT, Lillian Pliner, MD, Robin Streit, LMT, Nadine Jenkins, PhD, Bijal Parikh

Introduction: Nonpharmacological interventions like massage can play an important role in helping cancer patients manage cancer and cancer treatment-related symptoms. Many studies on massage for cancer patients have illustrated the positive impact, but it is unclear whether massage is routinely made available at cancer centers. The purpose of this project was to analyze if, where, and how massage was incorporated into cancer care in the Newark/New York City metropolitan area.

Methods: A mixed-methods analysis combined a content analysis of Web-based information with a telephone survey. Cancer centers within a 50 mile radius of the Rutgers Biomedical and Health Sciences Campus were chosen for review.

Results: Approximately half of the centers in the sample $(40,51.3 \%)$ offered massage therapy on-site for cancer patients undergoing treatment. An array of different massage modalities were offered to cancer patients including Reiki, reflexology, and Therapeutic Touch. Swedish massage, aromatherapy, manual lymphatic drainage, and neuromuscular massage were also referenced. Thirty-minute massages were the most frequently described length, with treatments ranging from 10 to 90 minutes. None of the centers billed insurance companies directly for massage therapy. Eighteen centers (23.1\%) employed or contracted with a licensed massage therapist. Patients had the choice to seek massage on their own, with only five centers $(6.4 \%)$ requiring approval of a physician. Massage treatment was recorded in the patient's medical record in only 11 centers $(14.1 \%)$.

Discussion: Findings reveal massage is only made available on-site at half the oncology centers in the Newark/New York City metropolitan area. Centers that did offer massage provided therapists credentialing to treat cancer patients. Since most massage services were free or paid out-of-pocket by patients, this suggests the impact of massage for cancer and cancer treatment-related symptoms is not stimulating recognition of massage as a viable supportive treatment by insurance companies.

\section{EFFECT OF DEEP CROSS-FRICTION MYOTHERAPY ON PRESSURE PAIN THRESHOLDS IN PATIENTS WITH NONSPECIFIC LOW BACK PAIN}

Andre Farasyn, PhD, PT, DO, R. Meeusen, PhD

Objective: To investigate (1) the pressure pain thresholds (PPTs) with respect to muscles related to 
low back pain (LBP) and to a muscle unrelated to lower back region, and (2) to explore the effect of deep cross-friction massage sessions [roptrotherapy] on PPTs, pain sensitivity, and disability.

Methods: 65 consecutive patients with subacute nonspecific LBP (nLBP) were allocated in this clinical trial. The primary outcome measures were the PPTs measured with the aid of a mechanical FISCHER algometer of levels L1, L3, and L5 of the Erector spinae and the Gluteus maximus. The middle of the left Triceps brachii was chosen as a neutral measuring point unrelated to LBP. Additionally, pain rating (VAS) and disability variables (ODI) were examined. In order to establish reference values, 64 healthy subjects [control group] were examined with respect to similar PPTs.

Results: The mean PPT values of the erector spinae and the gluteus maximus of the LBP group were significantly lower in comparison to the PPT values of the healthy group. The correlation between having LBP and PPT was highest at the L3 level of the erector spinae $[r=0.73, p<.001]$. The ODI and VAS decreased significantly after the 1 st roptrotherapy session by more than $50 \%$ and $25 \%$ after the second roptrotherapy session. At baseline, the PPTs of the erector spinae levels increased after the 3 roptrotherapy sessions, yielding a situation in which the patients no longer had back complaints, while the neutral triceps brachii remained unchanged over the entire time.

Conclusion: The results of this study demonstrate that patients with nLBP treated 3 times weekly with deep cross-friction massage, or roptrotherapy, had increased PPTs at the nLBP-related M. Erector spinae L1, L3, and L5 and M. Gluteus maximus levels and is probably responsible for the healing effect on local muscle tenderness. The 3-month follow-up results revealed that the PPT values of the nontreated and neutral Triceps brachii remained unchanged, while the PPT of the most highly nLBP related Erector spinae and Gluteus maximus levels increased in such way that the PPT values became similar to those of healthy subjects. Deep friction therapy of those muscle hardenings may desensitize central neural structures involved in pain perception and is not meaningfully influenced by the possible release of endogenous opioid hormones. The hypothesis that nonspecific LBP is primarily a myofascial pain syndrome caused by local injured muscular structures within the thoracolumbar spine and buttock should be supported.

\section{THE IMPACT OF THE SWEDISH MASSAGE ON THE KINESTHETIC DIFFERENTIATION IN HEALTHY INDIVIDUALS}

Mariusz P. Furmanek, Kamil Mustafa, Michal Pawlowski, Grzegorz Juras
Background: Swedish massage (in Europe also known as classic massage) is one of the common treatments that are used to provide optimal start and readiness of athletes. Kinesthetic differentiation (KD) is ability of an individual to assume the demanded muscle forces in order to optimize the required motor tasks (its economy and precision). There is no evidence as to how Swedish massage influences the kinesthetic differentiation. This issue is definitely worth exploring, taking into account the concept of a reflex-nervous activity of massage and the role of the kinesthetic differentiation in the structure of motor coordination.

Purpose: The main objective of the study was to evaluate the impact of the Swedish massage on the kinesthetic differentiation through measurements of maximal force grip and required partial force grip under static conditions.

Methods: Thirty participants took part in this investigation (17 women and 13 men). The subjects were $($ mean \pm SD): age: $22.2 \pm 1.09$ years old, height: $173.2 \pm 8.91 \mathrm{~cm}$, and weight: $68.5 \pm 13.48$ $\mathrm{kg}$. The assessment consisted of two kinesthetic differentiation (hand grip force) tests conducted on the dominant (DH) and nondominant hand (NDH). The first test reflected the natural state of $\mathrm{KD}$, while the second one was expected to reflect the impact of the 15-minute hand and forearm Swedish massage. These tests were done within 1 minute after the completion of massage. The procedure consisted of thirteen trials for each extremity. The first three were done for $100 \%$ of the participants' capabilities, which allowed to assess the participants' maximum force (Fmax), next five trials were done using $50 \%$ of maximum force (50\% of Fmax), and in the last five trials, the participants tried to use only $50 \%$ of their previous force $(1 / 2$ of $50 \%)$. Base on the model forces the absolute force production error (FPE) expressed in percentage was calculated for 50\% (FPE_50\%) and 25\% (FPE_25\%).

Results: The 2-way repeated measure analysis of variance ANOVA did not reveal any statistically significant changes in maximal force grip and kinesthetic differentiation between pre- and postmassage intervention in both dominant and nondominant hand. Correlations showed strong relationship between pre- and postmassage for maximum force $(r=0.92, p=0.01$ for $\mathrm{DH}$, and $r=$ $0.94, p=0.01$ for $\mathrm{NDH}$ ), and only for the FPE_50\% $(r=0.67, p=0.01$ for $\mathrm{DH}$, and $r=0.71, p=0.01$ for NDH).

Conclusions: The results obtained indicated that the application of the Swedish massage (for $15 \mathrm{~min}-$ utes in hand and forearm muscles) did not affect the kinesthetic differentiation in this particular young adults group. 


\section{BENEFITS OF MASSAGE THERAPY FOR PATIENTS WITH SEVERE BURN INJURIES: A CASE REPORT}

Robin Gawronski, LMT, Kristy Ruiz, LMT, Amanda Sonk, LMT, Travis Duffey, MS, LMT

Introduction: Although massage therapy (MT) is a less common treatment modality offered in inpatient pediatric hospital settings, research has shown that patients with severe burn injuries benefit from MT by having a decrease in pain, anxiety, and itching (Parlak Gürol et al., 2010). Research has also shown that when utilized prior to painful procedures, MT can lessen a patient's nonverbal pain and stress response, as well as facilitate dressing changes (Hernandez-Reif et al., 2001).

Objective: We present the case of a 13-year-old male who was admitted to the hospital after a selfinflicted flame burn, covering $83 \%$ of his total body surface area. This case study will demonstrate the benefits of including MT as part of an interdisciplinary burn team in order to improve range of motion (ROM), appearance of scarring, and decrease pain and anxiety over the course of treatment.

Methods: The patient received MT up to 10 times per week for sessions lasting from 30 minutes to two hours in individual or co-treat sessions with OT/PT to assist with increasing ROM and to decreasing pain and anxiety. ROM was recorded to demonstrate changes in pliability, and pictures were taken throughout his course in order to demonstrate changes in appearance of burn scars. Anxiety reduction was evidenced through changes in nonverbal indicators and heart rate values recorded using hospital monitors. Additionally, pain scales and verbal reports of itching were recorded throughout treatment.

Results: Throughout his admission, the patient displayed positive responses to MT including a decreased heart rate, reduced itchiness, improved appearance, and increased pliability of burn scars, and verbalized decrease in pain scale rating.

Conclusion: MT can be an integral part of the treatment course for severe burn sufferers. This case demonstrates the value of including MT throughout a patient's recovery to promote improved outcomes.

\section{"MIGRAINE — IS IT ALL IN THE HEAD?" A CASE REPORT ON BOWENWORK FOR MIGRAINE RELIEF*}

\author{
Sandra Gustafson, MHS, BSN, RN
}

\footnotetext{
* Gustafson S. "Migraine - Is It All In the Head?" A Case Report on Bowenwork for Migraine Relief. 2015. Manuscript submitted for publication.
}

Introduction: This study aimed to review and assess one migraineur's response to Bowenwork (gentle, soft-tissue bodywork technique) for reducing migraine occurrence and pain, pharmaceutical analgesic consumption, and improving the client's health-related quality of life (HRQoL), wellbeing, and activities of daily living (ADLs). Migraine is a complex neurological disorder characterized by episodic, neurogenic, cerebrovascular inflammation, and hypersensitization of brain-tissues and the central nervous system, causing severe pain and debility. Conventional treatments vary greatly and are often pharmaceutically based. Research literature points mostly to pharmaceutical prophylactic and symptomatic treatments, a few nonpharmaceutical, complementary, and alternative medicine (CAM) approaches, massage and bodywork studies, and no studies on Bowenwork for migraine intervention.

Participant: A 66-year-old Caucasian female with a history of debilitating migraine since childhood and severe neck pain resulting from 2 motor vehicle accidents (MVA) sustained as an adult.

Methodology: A descriptive observational casereport of one client's responses to receiving fourteen Bowenwork sessions, weekly to two-weekly, over a four-month period, using the self-reporting Measure Yourself Medical Outcome Profile version 2 (MYMOP2) to evaluate clinically meaningful changes. In previous studies involving participants with chronic pain conditions, MYMOP2 was considered a reliable and valid assessment tool. Prior to each Bowenwork session, data were recorded to track changes in migraine and neck pain occurrences, medication use, daily functional ability, and general sense of wellbeing. Specific Bowenwork procedures were applied in each session to address the client's symptoms.

Results: Over four months of receiving Bowenwork, the client progressively reported decreased migraine and neck pain; and by session 14, no further migraine, neck pain, nor medication use; and increased ADLs and wellbeing for 10 months thereafter.

Conclusion: Although limited to a single person, this case report suggests that Bowenwork may offer nonpharmaceutical migraine and pain relief for migraineurs; however, further research on larger populations is indicated.

\section{MASSAGE THERAPY FOR THE REDUCTION OF PAIN IN PALLIATIVE CARE PATIENTS AT A CHILDREN'S HOSPITAL}

Natalia Jaramillo, BA, Rika Meyer, PhD, Lauren Conn, BA, Jeffrey I. Gold, PhD

Introduction: Integrated health interventions, such as massage, are becoming more frequently used in 
pain management of palliative care patients (Mansky et al., 2006; Klick et al., 2010). However, there is limited research examining the effectiveness of massage in the reduction of pain among this population of patient.

Objective: The objective of this pilot study was to assess whether massage reduced pain intensity in children, adolescents, and young adults receiving palliative care.

Methods: We examined 11 palliative care patients at Children's Hospital Los Angeles (6 female; Ages $1-20 ; \mathrm{M}=15.18, \mathrm{SD}=5.74)$. Massage therapists completed a thirty-minute Swedish massage on each patient. Patients aged 8 years and above were asked to self-report their pain intensity before the massage, at present, and in the past week $n=10$ (Visual Analogue Scales (VAS) and (FACES Pain Scale-Revised, Hicks et al., 2001). Parents and nurses were asked openended questions about the overall effect of massage on the patient.

Results: Mean scores of pain decrease pre- $(\mathrm{M}=$ $3.25)$ to post-treatment $(\mathrm{M}=.50)$. Patients mean score of pain also decreased from past week $(M=4.57)$ to after massage $(\mathrm{M}=.57)$. However, paired sample $t$ tests were not significant for pain intensity of patients before and after massage $(\mathrm{t}(7)=2.11, p=.07)$ and pain in the past week and after massage $(\mathrm{t}(6)=2.37$, $p=.06)$. Analysis of pilot data suggests that massage may be effective in reducing self-reported pain intensity in children, adolescents, and young adults on the palliative care service. Qualitative data from parents and nurses also demonstrated that massage was beneficial in increasing relaxation and providing comfort to patients.

Conclusion: The study provides preliminary evidence supporting the effects of massage and its role in decreasing pain intensity and offering therapeutic value to children adolescents and young adults receiving palliative care. Further investigation is needed with a larger sample to assess pain reduction and other potential benefits.

\section{PERCEPTIONS OF EVIDENCE-BASED PRACTICE: A SURVEY OF MEMBERS OF THE FLORIDA STATE MASSAGE THERAPY ASSOCIATION}

Ronald J. Kettering, DHSc, MBA, Joan S. Leafman, $\mathrm{PhD}$, Lisa A. Wallace, PhD, Jerrilyn A. Cambron, $\mathrm{PhD}, \mathrm{DC}$

Introduction: Massage therapists must have the necessary knowledge and skills to evaluate available research to make effective clinical decisions. However, the extent to which the attitudes of massage therapists towards the value of using evidence in patient care, and their confidence in their ability to implement evidence-based practice (EBP), is lacking in the body of available evidence.

Purpose: This study explored the perceptions of the clinical value of EBP among members of the Florida State Massage Therapy Association (FSMTA) and their confidence in implementing it in practice.

Methods: Members $(\mathrm{N}=498)$ completed a secure online survey related to beliefs and attitudes about EBP and met the research inclusion criteria. This study used univariate nonparametric statistics to analyze demographic and EBP variables.

Results: The findings suggest participants perceived EBP as an important aspect of practicing massage therapy. Statistically significant findings included: positive belief in the importance of critical appraisals $(68.0 \%, \mathrm{n}=259)$ and belief that EBP can improve patient care outcomes $(66.4 \%$, $\mathrm{n}=253$ ). However, there remains a need among massage therapists to develop confidence in using EBP to optimize clinical outcomes. The belief that EBP is difficult to utilize in clinical practice was significantly negative $(93.2 \%, \mathrm{n}=355)$. Confidence in using evidence to answer clinical questions $(52.8 \%, \mathrm{n}=201)$ and confidence in the ability to overcome EBP implementation barriers $(52.0 \%, \mathrm{n}$ $=198$ ) were low.

Conclusion: This study described important EBP and confidence attributes of the population of FSMTA members, and provided important foundational data that can be used to help in the development of future massage therapy research literacy programs and clinical application policies.

\section{USING DYNAMIC ANGULAR PETRISSAGE FOR AXILLARY WEB SYNDROME OCCURRING AFTER BREAST CANCER SURGERY}

\author{
Paul A. Lewis, RMT, CDT, Joan E. Cunningham, PhD
}

Introduction: Axillary web syndrome (AWS), also called lymphatic cording, typically presents in the weeks after axillary surgery for breast cancer. This painful condition is likely angiolymphatic and fibrotic in origin, and restricts upper extremity range of motion (ROM). It has no established treatment, although physical therapy and other approaches have been used to variable effect.

Objective: Swedish massage and specialized passive movement techniques were applied in the case of a young woman with axillary cording, to investigate effectiveness in reducing pain and restricted ROM caused by this condition.

Case Presentation and Methods: A female patient who had recently undergone axillary surgery presented with pain (self-reported as 5 on a scale of $0-10$ ) and restricted use of the ipsilateral upper extremity. Extent of cording (taut, from axilla to wrist) and flexion at 
glenohumeral joint $\left(\mathrm{GH} ; 140^{\circ}\right.$ measured by goniometer) were assessed. Therapeutic massage was administered over two sessions. Methods included dynamic angular petrissage techniques: stretching and relaxing the target tissue by taking the limb through all possible angles of movement while simultaneously and segmentally applying Swedish and non-Swedish (including myofascial, lymphatic drainage, etc.) techniques to the underlying soft tissue. The cord was considered a structure to be released, rather than a tissue to be torn or broken. The patient practiced prescribed self-care exercises between treatment sessions.

Results: After Session One, pain was reduced to $0 / 10$, GH flexion improved to $170^{\circ}$, and cording was visibly reduced. After Session Two, the cord was only residually apparent, with no ROM restrictions even during hyperextension. Long-term outcome was complete resolution after only two sessions, with no recurrence of AWS.

Conclusions: Pain, restricted flexion, and cording were quickly and gently eliminated. The combination of massage and movement using dynamic angular petrissage techniques is proposed as an effective, efficient treatment approach for axillary web syndrome.

\section{A DESCRIPTIVE STUDY OF THE MYOFASCIAL UNWINDING EXPERIENCE}

\section{Rebecca S. Massmann, LMT}

Introduction: Myofascial unwinding (MU) describes involuntary movement and sensations in the body occurring in response to a practitioner's touch, or through self-induction. Anecdotal accounts of the MU experience exist; however, there is a paucity of literature examining the experience of $\mathrm{MU}$ for the recipient.

Purpose: The aim of this study was to generate a rich description of recipients' MU experience.

Methods: Naturalistic inquiry and descriptive content analysis guided the research. Data were collected through semistructured interviews. The purposive sample consisted of eight highly experienced practitioners of manual therapies, who were also experienced recipients of MU.

Findings: The findings portray the essential features of the MU experience:

- Preconditions for the MU experience include feeling safe in order to let go, working with a trusted practitioner, and trusting oneself in the process.

- The practitioner-recipient relationship influences the MU experience.

- The lived experience of MU can include a wide range of physiological responses, emotional expression, mental processes, and transpersonal experiences.
- The impact of the MU experience for recipients is highly individualized and multidimensional. Outcomes reflected in this study demonstrated that MU supports the body's ability to heal and prevent injuries, process traumatic events, support the aging process, improve physiological function, manage emotions, and affect changes in personal belief systems and patterns of behavior.

Implications: The use of an empathic practitioner and the body-centered approach to MU, along with the multidimensional experience and psychophysiological responses to MU, suggest that it may be effective as an intervention in trauma recovery.

Conclusion: Myofascial unwinding is a process that is centered in trusting the inherent wisdom of the body to inform the psychophysiological, psychosocial, and/or transpersonal levels necessary to promote a greater state of ease for the recipient.

\section{COMPARING THE EFFECTS OF REST AND MASSAGE FOLLOWING SUBMAXIMAL AEROBIC EXERCISE*}

\section{Portia B. Resnik, MA, ATC, LMT}

Introduction: Postexercise massage can be utilized to help promote recovery from exercise on the cellular level, as well as systemically, by increasing parasympathetic activity. No studies to date have been done to assess the effects of massage on postexercise metabolic changes, including excess postexercise oxygen consumption (EPOC). The purpose of this study was to compare the effects of massage recovery and resting recovery on a subject's heart rate variability and selected metabolic effects following a submaximal treadmill exercise session.

Methods: One healthy 24-year-old female subject performed 30 minutes of submaximal treadmill exercise prior to resting or massage recovery sessions. Metabolic data were collected throughout the exercise sessions and at three 10-minute intervals postexercise. Heart rate variability was evaluated for 10 minutes after each of two 30-minute recovery sessions, either resting or massage.

Results: Heart rate returned to below resting levels (73 bpm) with 30 and 60 minutes of massage recovery (72 bpm and $63 \mathrm{bpm}$, respectively) compared to 30 and 60 minutes of resting recovery $(77 \mathrm{bpm}$ and 74 bpm, respectively). Heart rate variability data showed a more immediate shift to the parasympathetic state following 30 minutes of massage $(1.152 \mathrm{LF} / \mathrm{HF}$

* Resnik P. Comparing the Effects of Rest and Massage Following Submaximal Aerobic Exercise. 2015. Manuscript submitted for publication. 
ratio) versus the 30-minute resting recovery (6.91 $\mathrm{LF} / \mathrm{HF}$ ratio). It took 60 minutes of resting recovery to reach similar heart rate variability levels (1.216 $\mathrm{LF} / \mathrm{HF}$ ) found after 30 minutes of massage. Ventilations after 30 minutes of massage recovery averaged $7.1 \mathrm{bpm}$ compared to $17.9 \mathrm{bpm}$ after 30 minutes of resting recovery.

Discussion: No differences in EPOC were observed through either the resting or massage recovery based on the metabolic data collected. Massage can be used to help an athlete shift into parasympathetic activity more quickly than rest alone following a submaximal exercise session.

\section{UNDERSTANDING INSURANCE REIMBURSEMENT GUIDELINES FOR MASSAGE THERAPY}

\section{Robin Streit, LMT, Virginia S. Cowen, PhD}

Introduction: Massage therapy is one of the most common nonconventional therapies for orthopedic conditions and is within the scope of many providers. Physical therapists, occupational therapists, and chiropractors frequently receive insurance reimbursement for manual therapy, but it is unclear how the insurance policies factor the provider's qualifications into payment. This study aims to understand general insurance reimbursement guidelines for massage therapy, specifically as it pertains to the providers, techniques, and covered conditions.

Methods: A list of US health insurance companies was generated from A.M. Best's online database. All of the companies with policies $(\mathrm{N}=26)$ available in more than one eastern state were chosen for review. Each carrier's website was searched for policy information with terms "massage therapy" and "manual therapy." Information about massage techniques, providers, conditions, and practice guidelines was coded and analyzed using descriptive statistics.

Results: All cases offered massage therapy administered by a massage therapist through a discounted rate program. Most companies covered massage, but the extent of coverage was limited to plan type. Reimbursable techniques included Swedish, manual lymphatic drainage, mobilization, myofascial release, and traction. In most cases, chiropractors, physical therapists, and occupational therapists could bill for massage services directly. Few cases required massage therapists to be employed and/or supervised by a doctor. Reimbursement was typically limited to 15-minute increments, covered as one part of a comprehensive rehabilitation plan, and needed to be medically necessary (prescribed by a doctor). Massage was commonly covered for neuromusculoskeletal conditions.
Discussion: US health insurance policies tend to cover massage, but limit reimbursement to doctors. The strict practice guidelines appear to disregard laws governing massage therapists. Future research should specifically compare the guidelines, laws, and findings across states to identify inconsistencies. Coverage inclusion will heighten acceptance by other health care professionals and strengthen the profession's contribution to the health care system.

\section{EFFECTIVENESS OF MASSAGE THERAPY ON PEDIATRIC PATIENTS WITH CHRONIC PAIN}

\author{
Ling Tsui, Arianna Robin
}

Introduction: Massage therapy (MT) is a common intervention used in patients with chronic pain. Although most literature on the effects of MT focuses on adults, existing studies on pediatric MT have shown decreases in pain and distress immediately after each treatment. This study examined the overall benefits of massage in pediatric patients with chronic pain across 4 treatments.

Methods: Fifty-one participants (74.5\% female and $23.5 \%$ male; ages 5 to $19 ; \mathrm{M}$ age $=15, \mathrm{SD}=3.21$ ) from an urban children's hospital-based pediatric pain clinic completed a survey pre- and postmassage and self-reported a variety of critical outcomes. Anxiety, comfort, relaxation, and calmness were assessed using a $10 \mathrm{~cm}$ Visual Analog Scales (VAS) ranging from 0 (strongly disagree) to 10 (strongly agree); pain intensity was evaluated using the Faces Pain Scale-Revised (FPS-R) (Hicks et al., 2001), and sleepiness was assessed with the Epworth Sleepiness Scale (Johns, 1991).

Results: Results revealed significant changes in comfort $(\mathrm{F}(3,42)=5.89, p=.02)$, relaxation $(\mathrm{F}(3,54)=6.27, p=.00)$, and calmness $(\mathrm{F}(3,45)=$ $2.18, p=.00)$, indicating that MT is effective in increasing comfort, relaxation and calmness in patients across time. Although significant changes in pain were found from pre- to post- within each treatment, no significant changes in anxiety, pain VAS, Pain FPS-R, sleepiness across treatments were reported over time, suggesting that MT's effect in reducing anxiety, pain, and sleepiness is temporary, as opposed to long-lasting improvements.

Conclusion: Future studies should 1) include a larger sample; 2) provide more than four sessions to examine the ongoing cumulative effects of MT versus the acute pain relief; and 3) continue to investigate the effectiveness of MT alone and in combination with other integrative health interventions. Integrated health interventions are critical in the management of patients with chronic health conditions. 


\section{DEVELOPMENT OF A HOSPITAL-BASED MASSAGE THERAPY COURSE AT AN ACADEMIC MEDICAL CENTER*}

\section{Lisa Dion}

Background: Massage therapy is offered increasingly in US medical facilities. Although the United States has many massage schools, their education differs, along with licensure and standards. As massage therapy in hospitals expands and proves its value, massage therapists need increased training and skills in working with patients who have various complex medical concerns, to provide safe and effective treatment. These services for hospitalized patients can impact patient experience substantially, and provide additional treatment options for pain and anxiety, among other symptoms. This poster summarizes the initial development and description of a hospital-based massage therapy course at a Midwest medical center.

Methods: A hospital-based massage therapy course was developed on the basis of clinical experience and knowledge from massage therapists working in the complex medical environment. This massage therapy course has three components in its educational experience: online learning, classroom study, and a 25-hour shadowing experience. The in-classroom study portion includes an entire day in the simulation center.

Results: The hospital-based massage therapy course addresses the educational needs of therapists transitioning to work with interdisciplinary medical teams and with patients who have complicated medical conditions. Feedback from students in the course has indicated key learning opportunities and additional content that is needed to address the knowledge and skills necessary when providing massage therapy in a complex medical environment.

Conclusions: The complexity of care in medical settings is increasing while the length of hospital stay is decreasing. For this reason, massage provided in the hospital requires more specialized training to work in these environments. This poster provides an example of the initial steps in how to address some of the educational needs of therapists who are transitioning to working in the complex medical environment.

* Dion LJ, Cutshall SM, Rodgers NJ, Hauschulz JL, Dreyer NE, Thomley BS, Bauer B. Development of a Hospital-based Massage Therapy Course at an Academic Medical Center. Int J Therapeutic Massage and Bodywork. 2015;8(1):25-30. 